$\mathbb{T}$ periodica polytechnica

\author{
Electrical Engineering \\ and Computer Science \\ $56 / 1(2012) 29,34$ \\ doi: 10.3311/PPee.7165 \\ http://periodicapolytechnica.org/ee \\ Creative Commons Attribution (1)
}

RESEARCH ARTICLE

\section{Prediction based - high frequency trading on financial time series}

János Levendovszky / Farhad Kia

Received 2011-11-12, accepted 2012-09-22

\begin{abstract}
In this paper we investigate prediction based trading on financial time series assuming general $A R(J)$ models. A suitable nonlinear estimator for predicting the future values will be provided by a properly trained FeedForward Neural Network (FFNN) which can capture the characteristics of the conditional expected value. In this way, one can implement a simple trading strategy based on the predicted future value of the asset price and comparing it to the current value. The method is tested on FOREX data series and achieved a considerable profit on the mid price. In the presence of the bid-ask spread, the gain is smaller but it still ranges in the interval 2-6 percent in 6 months without using any leverage. FFNNs can provide fast prediction which can give rise to high frequency trading on intraday data series.
\end{abstract}

\section{Keywords \\ algorithmic trading $\cdot$ time series analysis}

\section{János Levendovszky}

Department of Networked Systems and Services, BME, H-1117 Budapest, Magyar tudósok körútja 2., Hungary

e-mail: levendov@hit.bme.hu

\section{Farhad Kia}

Department of Networked Systems and Sevices, BME, H-1117 Budapest, Magyar tudósok körútja 2.

\section{Introduction}

In the advent high speed computation and ever increasing computational power, algorithmic trading has been receiving a considerable interest [1-4]. The main focus of research is to develop real-time algorithms which can cope with portfolio optimization and price estimation within a very small time interval enabling high frequency, intraday trading. In this way, fast identification of favorable patterns on time series becomes feasible on small time scales which can give rise to profitable trading where asset prices follow each other in sec or msec range.

Several papers have been dealt with algorithmic trading by using fast prediction algorithms [5-7] or by identifying mean reverting portfolios [7]-9]. The paper [10] uses linear prediction which however proves to be poor to capture the complexity of the underlying time series. Other methods [8,9] are focused on identifying mean reverting portfolios and launch a trading action (e.g. buy) if the portfolio is out of the mean and taking the opposite action when it returns to the mean.

In our approach, we focus on prediction based trading by estimating the future price of the time series by using a nonlinear predictor in order to capture the underlying structure of the time series. The investigated time series can either refer to foreign exchange rates, single asset prices or the value of a previously optimized portfolio. By using FFNNs, which exhibit universal representation capabilities, one can model the nonlinear $\operatorname{AR}(J)$ process (the current value of the time series depends on J previous values and corrupted by additive Gaussian noise). Assuming the price series to be a nonlinear $\operatorname{AR}(J)$ process, we first develop the optimal trading strategy and then approximate the parameters of nonlinear $\mathrm{AR}(\mathrm{J})$ by an FFNN.

In this way, one can obtain a fast adaptive trading procedure which, in the first stage, runs a learning algorithm for parameter optimization based on some observed prices and then, in the second stage, provides near optimal estimation of future prices. The numerical results obtained on Forex rates have demonstrated that the method is profitable and achieves more than $1 \%$ profit in one month with leverage 1:1 which can be much bigger if we use leverage.

The paper treats this material in the following structure: 
- in Section 2 the model is outlined;

- in Section 3 the optimal strategy is derived first for trading on midprices and then it is extended to trading in the presence of bid-ask spread;

- in Section 5 numerical results and performance analysis is given;

- in Section 7 some conclusions are drawn.

\section{The model}

Let us assume that we trade on the mid prices and the corresponding asset price series is denoted by $x_{k}$ and follows a nonlinear $\operatorname{AR}(J)$ process

$$
x_{k}=F\left(x_{k-1}, \ldots, x_{k-J}\right)+v_{k}
$$

where $F$ is a Borel measurable function and $v_{k} \sim N(0, \sigma)$ i.i.d.r.v.-s, being independent of $x_{k}$. For trading, we construct an estimator

$$
\tilde{x}_{k}=\operatorname{Net}\left(x_{k-1}, \ldots, x_{k-J}, w_{1}, \ldots, w_{M}\right)=\operatorname{Net}(\mathbf{x}, \mathbf{w})
$$

where

$$
\begin{aligned}
& \tilde{x}_{k}=\operatorname{Net}(\mathbf{x}, \mathbf{w}) \\
&=\varphi\left(\sum_{i} w_{i}^{(L)} \varphi\left(\sum_{j} w_{i j}^{(L-1)} \ldots \varphi\left(\sum_{m} w_{n m}^{(1)} x_{k-m}\right) \ldots\right)\right)
\end{aligned}
$$

is a Feedforward NeuralNetwork (FFNN) depicted by Figure 1 and vector $\mathbf{w}$ denotes the free parameters subject to training.

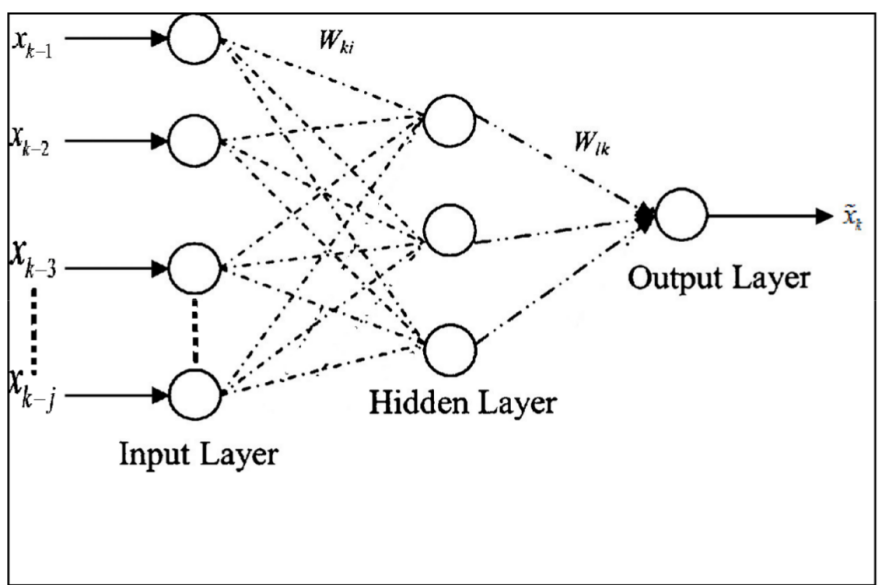

Fig. 1. The structure of feed forward neural network(FFNN)

Trading is performed as follows:

- Stage 1. Observing a historical time series and forming a training set

$$
\tau^{(K)}=\left\{\left(\mathbf{x}^{(k)}, x_{k}\right), k=1, \ldots, K\right\}
$$

where $\mathbf{x}^{(k)}=\left(x_{k-J}, \ldots, x_{k-1}\right)$.
- Stage 2. Training the weights by minimizing the objective function

$$
\min _{w} \frac{1}{K}\left(x_{k}-\operatorname{Net}\left(\mathbf{x}^{(k)}, \mathbf{w}\right)\right)^{2}
$$

by the Back Propagation (BP) algorithm.

- Stage 3. Trading on the real data as follows:

Calculate $\tilde{x}_{k}=\operatorname{Net}\left(\mathbf{x}^{(k)}, \mathbf{w}\right)$, where $\mathbf{x}^{(k)}=\left(x_{k-J}, \ldots, x_{k-1}\right)$ and we are in time instant $k-1$.

$$
\begin{aligned}
& \text { if } \tilde{x}_{k}>x_{k-1} \text { then buy at time instant } k-1 \\
& \text { and sell at time instant } k \\
& \text { if } \tilde{x}_{k}<x_{k-1} \text { then sell at time instant } k-1 \\
& \text { and buy back at time instant } k
\end{aligned}
$$

\section{The optimality of the proposed trading strategy}

In this section we prove the optimality of the strategy outlined above.

Since

$$
\lim _{K \rightarrow \infty} \frac{1}{K}\left(x_{k}-\operatorname{Net}\left(\mathbf{x}^{(k)}, \mathbf{w}\right)\right)^{2}=E\left(x_{k}-\operatorname{Net}\left(\mathbf{x}^{(k)}, \mathbf{w}\right)\right)^{2}
$$

thus

$$
\operatorname{Net}\left(\mathbf{x}^{(k)}, \mathbf{w}_{\text {opt }}\right)=E\left(x_{k} \mid \mathbf{x}^{(k)}\right)=F\left(x_{k-1}, \ldots, x_{k-J}\right)
$$

taking into account that $E\left(v_{k}\right)=0$. Note, that the FFNN can represent $E\left(x_{k} \mid \mathbf{x}^{(k)}\right)=F\left(x_{k-1}, \ldots, x_{k-J}\right)$ as being a universal approximator in $L^{p}$ spaces. It is also clear that the best trading decision which maximizes the return is given as

$$
\begin{aligned}
& \text { If } P\left(x_{k}>x_{k-1}\right)>P\left(x_{k}<x_{k-1}\right) \\
& \text { then buy at } \mathrm{k}-1 \text { and sell at } \mathrm{k} \\
& \text { If } P\left(x_{k}>x_{k-1}\right)>P\left(x_{k}<x_{k-1}\right) \\
& \text { then sell at } \mathrm{k}-1 \text { and buy at } \mathrm{k}
\end{aligned}
$$

Let us now investigate $P\left(x_{k}>x_{k-1}\right)$

$$
\begin{aligned}
P\left(x_{k}>x_{k-1}\right) & =P\left(F\left(x_{k-1}, \ldots, x_{k-J}\right)+v_{k}>x_{k-1}\right) \\
& =P\left(v_{k}>x_{k-1}-F\left(x_{k-1}, \ldots, x_{k-J}\right)\right) \\
& =P\left(v_{k}>x_{k-1}-\operatorname{Net}\left(\mathbf{x}^{(k)}, \mathbf{w}_{\text {opt }}\right)\right) \\
& =P\left(v_{k}>x_{k-1}-\tilde{x}_{k}\right) \\
& =1-\Phi\left(\frac{x_{k-1}-\tilde{x}_{k}}{\sigma}\right)=\frac{1}{\sigma \sqrt{2 \pi}} \int_{x_{k-1}-\tilde{x}_{k}}^{\infty} e^{-\frac{u^{2}}{2}} d u
\end{aligned}
$$

Due to the symmetric nature of the p.d.f. of $v_{k}$ one can see that if $\tilde{x}_{k}>x_{k}$ then

$$
P\left(v_{k}>x_{k-1}-\tilde{x}_{k}\right)>P\left(v_{k}<x_{k-1}-\tilde{x}_{k}\right)
$$

As a result, the trading strategy (3) is indeed optimal.

Based on the discussion above, one can implement the prediction based training according to the following steps:

1 Gather data from the training set

$$
\tau^{(k)}=\left\{\left(x_{k}, \mathbf{x}^{(k)}\right), k=1, \ldots, K\right\}
$$


2 Choose a proper FFNN (for complexity issues see [11] )

3 Train the weights of the FFNN by performing the error back propagation algorithm.

$$
\min _{\mathbf{w}} \frac{1}{K}\left(x_{k}-\operatorname{Net}\left(\mathbf{x}^{(k)}, \mathbf{w}\right)\right)^{2}
$$

4 Implement the trading strategy

$$
\begin{aligned}
& \text { if } \tilde{x}_{k}>x_{k-1} \text { then buy at time instant k-1 } \\
& \text { and sell at time instant k } \\
& \text { if } \tilde{x}_{k}<x_{k-1} \text { then sell at time instant k-1 } \\
& \text { and buy back at time instant } \mathrm{k}
\end{aligned}
$$

\section{Extension to Bid-Ask spread}

In the presence of bid ask spread we have two time series, ask price $y^{(k)}=\left(y_{k-J}, \ldots, y_{k-1}\right)$ and bid price $x^{(k)}=\left(x_{k-J}, \ldots, x_{k-1}\right)$ with $y^{(k)}>x^{(k)}$.

Based in the previous discussion we assume that

$$
x_{k}=F\left(y_{k}, \ldots, y_{k-J}, x_{k-1}, \ldots, x_{k-J}\right)+v_{k}
$$

and

$$
y_{k}=G\left(y_{k}, \ldots, y_{k-J}, x_{k-1}, \ldots, x_{k-J}\right)+\eta_{k}
$$

where $F$ and $G$ are Borel measurable functions and $v_{k} \sim N(0, \sigma)$ and $\eta_{k} \sim N(0, \sigma)$, respectively.

As was seen before, profit can be achieved by pursuing the following strategy

$$
\begin{aligned}
& \text { If } P\left(x_{k}>y_{k-1}\right)>P\left(x_{k}<y_{k-1}\right) \\
& \text { then buy at } \mathrm{k}-1 \text { and sell at } \mathrm{k} \\
& \text { If } P\left(y_{k}<x_{k-1}\right)<P\left(y_{k}>x_{k-1}\right) \\
& \text { then sell at k-1 and buy at } \mathrm{k}
\end{aligned}
$$

One can deploy two FFNNs to predict the current values of time series $y_{k}$ and $x_{k}$, as follows

$$
\begin{aligned}
\tilde{x}_{k} & =\operatorname{Net}^{(B i d)}\left(y_{k-1}, \ldots, y_{k-J}, x_{k-1}, \ldots, x_{k-J}, w_{1}, \ldots, w_{M}\right) \\
& =\operatorname{Net}^{(B i d)}(\mathbf{y}, \mathbf{x}, \mathbf{w}) \\
\tilde{y_{k}} & =\operatorname{Net}^{(A s k)}\left(y_{k-1}, \ldots, y_{k-J}, x_{k-1}, \ldots, x_{k-J}, u_{1}, \ldots, u_{M}\right) \\
& =\operatorname{Net}^{(B i d)}(\mathbf{y}, \mathbf{x}, \mathbf{u})
\end{aligned}
$$

where training can take place by observing a historical time series and forming the training sets

$$
\tau^{(A s k)}=\left\{\left(\mathbf{y}^{(k)}, \mathbf{x}^{(k)}, y_{k}\right), k=1, \ldots, K\right\}
$$

and

$$
\tau^{(B i d)}=\left\{\left(\mathbf{y}^{(k)}, \mathbf{x}^{(k)}, x_{k}\right), k=1, \ldots, K\right\}
$$

where

$$
\begin{gathered}
\mathbf{y}^{(k)}=\left(y_{k-J}, \ldots, y_{k-1}\right) \text { and } \\
\mathbf{x}^{(k)}=\left(x_{k-J}, \ldots, x_{k-1}\right)
\end{gathered}
$$

and training the weights by back propagation according to the objective functions

$$
\min _{w} \frac{1}{K}\left(x_{k}-\operatorname{Net}^{(B i d)}\left(\mathbf{y}^{(k)}, \mathbf{x}^{(k)}, \mathbf{w}\right)\right)^{2}
$$

and

$$
\min _{u} \frac{1}{K}\left(x_{k}-\operatorname{Net}^{(A s k)}\left(\mathbf{y}^{(k)}, \mathbf{x}^{(k)}, \mathbf{u}\right)\right)^{2}
$$

Let us investigate the optimality of the trading strategy. Again, gain can be accumulated

$$
\begin{aligned}
& \text { If } P\left(x_{k}>y_{k-1}\right)>P\left(x_{k}<y_{k-1}\right) \\
& \text { then buy at } \mathrm{k}-1 \text { and sell at } \mathrm{k} \\
& \text { If } P\left(y_{k}<x_{k-1}\right)<P\left(y_{k}>x_{k-1}\right) \\
& \text { then sell at } \mathrm{k}-1 \text { and buy at } \mathrm{k}
\end{aligned}
$$

Furthermore, it holds that

$$
\begin{aligned}
& \lim _{K \rightarrow \infty} \frac{1}{K}\left(x_{k}-\operatorname{Net}^{(b i d)}\left(\mathbf{y}^{(k)}, \mathbf{x}^{(k)}, \mathbf{w}\right)\right)^{2} \\
&=E\left(x_{k}-\operatorname{Net}^{(b i d)}\left(\mathbf{y}^{(k)}, \mathbf{x}^{(k)}, \mathbf{w}\right)\right)^{2} \\
&=E\left(x_{k} \mid \mathbf{y}^{(k)}, \mathbf{x}^{(k)}\right)=F\left(\mathbf{y}^{(k)}, \mathbf{x}^{(k)}\right) \\
& \lim _{K \rightarrow \infty} \frac{1}{K}\left(y_{k}-\operatorname{Net}^{(a s k)}\left(\mathbf{y}^{(k)}, \mathbf{x}^{(k)}, \mathbf{w}\right)\right)^{2} \\
&=E\left(y_{k}-\operatorname{Net}^{(a s k)}\left(\mathbf{y}^{(k)}, \mathbf{x}^{(k)}, \mathbf{w}\right)\right)^{2} \\
&=E\left(y_{k} \mid \mathbf{y}^{(k)}, \mathbf{x}^{(k)}\right)=G\left(\mathbf{y}^{(k)}, \mathbf{x}^{(k)}\right)
\end{aligned}
$$

Let us evaluate the probability $P\left(x_{k}>y_{k-1}\right)$

$$
\begin{aligned}
P\left(x_{k}>y_{k-1}\right) & =P\left(F\left(y_{k-1}, \ldots, y_{k-J}, x_{k-1}, \ldots, x_{k-J}\right)+v_{k}>y_{k-1}\right) \\
& =P\left(v_{k}>y_{k-1}-F\left(y_{k-1}, \ldots, y_{k-J}, x_{k-1}, \ldots, x_{k-J}\right)\right) \\
& =P\left(v_{k}>y_{k-1}-\operatorname{Net}\left(\mathbf{y}^{(k)}, \mathbf{x}^{(k)}, \mathbf{w}_{o p t}\right)\right. \\
& =P\left(v_{k}>y_{k-1}-\tilde{x}_{k}\right) \geq P\left(v_{k}>x_{k-1}-\tilde{x}_{k}\right)
\end{aligned}
$$

Due to the symmetric nature of the p.d.f. of $v_{k}$ one can see that if $\tilde{x}_{k}>x_{k}$ then

$$
\begin{aligned}
P\left(v_{k}>y_{k-1}-\tilde{x}_{k}\right) \geq & P\left(v_{k}>x_{k-1}-\tilde{x}_{k}\right)> \\
& P\left(x v_{k}<x_{k-1}-\tilde{x}_{k}\right) \geq P\left(v_{k}<y_{k-1}-\tilde{x}_{k}\right)
\end{aligned}
$$

A very similar proof can be given for the other case.

As a result, the optimal trading strategy is as follows

$$
\begin{aligned}
& \text { if } \tilde{x}_{k}>y_{k-1} \\
& \text { then buy at time instant } \mathrm{k}-1 \\
& \text { and sell at time instant } \mathrm{k} \\
& \text { if } \tilde{y}_{k}<x_{k-1} \\
& \text { then sell at time instant } \mathrm{k}-1 \\
& \text { and buy back at time instant } \mathrm{k}
\end{aligned}
$$

while the whole process can be implemented as follows:

1 Observe historical data and form training sets

$$
\tau_{\text {Ask }}^{(k)}=\left\{\left(\mathbf{y}^{(k)}, \mathbf{x}^{(k)}, y_{k}\right), k=1, \ldots, K\right\}
$$


and

$$
\tau_{\text {Bid }}^{(k)}=\left\{\left(\mathbf{y}^{(k)}, \mathbf{x}^{(k)}, x_{k}\right), k=1, \ldots, K\right\}
$$

where $\mathbf{y}^{(k)}=\left(y_{k-J}, \ldots, y_{k-1}\right)$ and $\mathbf{x}^{(k)}=\left(x_{k-J}, \ldots, x_{k-1}\right)$

2 Construct FFNNs

$$
\begin{aligned}
\tilde{x}_{k}=\mathrm{Net}^{(B i d)}\left(y_{k-1}, \ldots, y_{k-J}, x_{k-1}, \ldots, x_{k-J},\right. \\
\left.w_{1}, \ldots, w_{M}\right)=\operatorname{Net}^{(B i d)}(\mathbf{y}, \mathbf{x}, \mathbf{w})
\end{aligned}
$$

and

$$
\begin{aligned}
& \tilde{y}_{k}=\operatorname{Net}^{(A s k)}\left(y_{k-1}, \ldots, y_{k-J}, x_{k-1}, \ldots, x_{k-J},\right. \\
&\left.u_{1}, \ldots, u_{M}\right)=\operatorname{Net}^{(B i d)}(\mathbf{y}, \mathbf{x}, \mathbf{u})
\end{aligned}
$$

3 Train the FNNs by BP algorithm to minimize the following error

and

$$
\min _{w} \frac{1}{K}\left(x_{k}-\operatorname{Net}^{(B i d)}\left(\mathbf{y}^{(k)}, \mathbf{x}^{(k)}, \mathbf{w}\right)\right)^{2}
$$

$$
\min _{u} \frac{1}{K}\left(x_{k}-\operatorname{Net}^{(A s k)}\left(\mathbf{y}^{(k)}, \mathbf{x}^{(k)}, \mathbf{u}\right)\right)^{2}
$$

4 In the current stage of the process use the estimates

$$
\begin{aligned}
\tilde{x}_{k}=\operatorname{Net}^{(B i d)}\left(y_{k-1}, \ldots, y_{k-J}, x_{k-1}, \ldots, x_{k-J},\right. \\
\left.w_{1}, \ldots, w_{M}\right)=\operatorname{Net}^{(B i d)}(\mathbf{y}, \mathbf{x}, \mathbf{w})
\end{aligned}
$$

and

$$
\begin{aligned}
\tilde{y}_{k}=\operatorname{Net}^{(A s k)}\left(y_{k-1}, \ldots, y_{k-J}, x_{k-1}, \ldots, x_{k-J},\right. \\
\left.u_{1}, \ldots, u_{M}\right)=\operatorname{Net}^{(B i d)}(\mathbf{y}, \mathbf{x}, \mathbf{u})
\end{aligned}
$$

5 Trade by the rules

$$
\begin{aligned}
& \text { if } \tilde{x}_{k}>y_{k-1} \\
& \text { then buy at time instant } \mathrm{k}-1 \\
& \text { and sell at time instant } \mathrm{k} \\
& \text { if } \tilde{y}_{k}<x_{k-1} \\
& \text { then sell at time instant } \mathrm{k}-1 \\
& \text { and buy back at time instant } \mathrm{k}
\end{aligned}
$$

\section{Numerical results}

We have tested the proposed method in three different cases:

- in the first case we predict the future price based on mid-price and we also trade on mid-price;

- in the second case we still predict by using the mid-price but we trade in the presence of Bid/Ask spread;

- finally, in the last case, we predict by using the Bid/Ask and trade on Bid/Ask.

The testing parameters (period length, timeframe $\mathbf{J}$, initial deposit, ...) are the same in all 3 cases and taken to be: period length = 1 Month (2012.06.01-2012.07.02); J=5; training period=20; timeframe=M15; currency : EURUSD(FOREX REAL DATA); initial deposit $=1000$

In the figures the number of trades is shown in the horizontal axis, while the account balance is indicated on the vertical axis.

\subsection{Prediction and trading on mid price}

As was mentioned before, in the first case we work only predict and trade on the mid-price. The results are indicated by figures 2 and 3 , respectively.

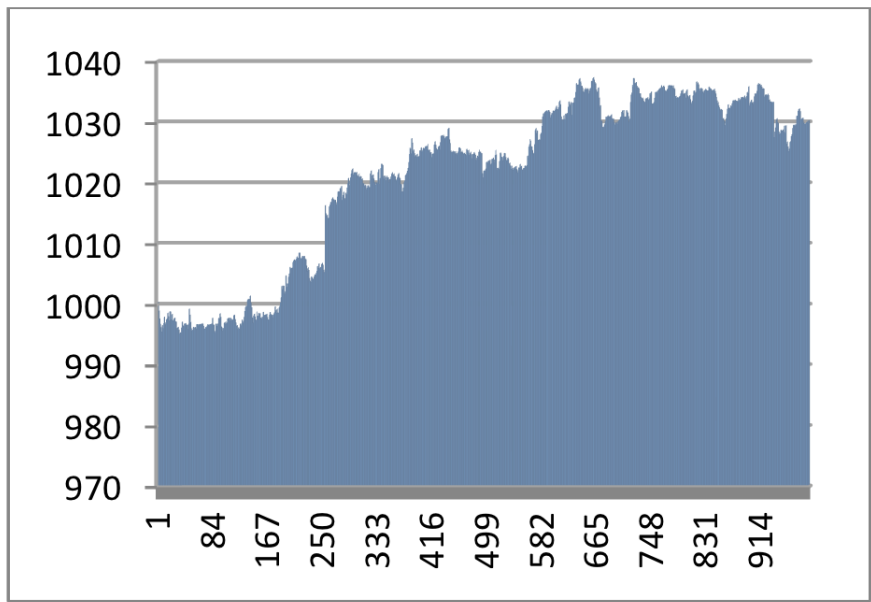

Fig. 2. Balance with respect to time

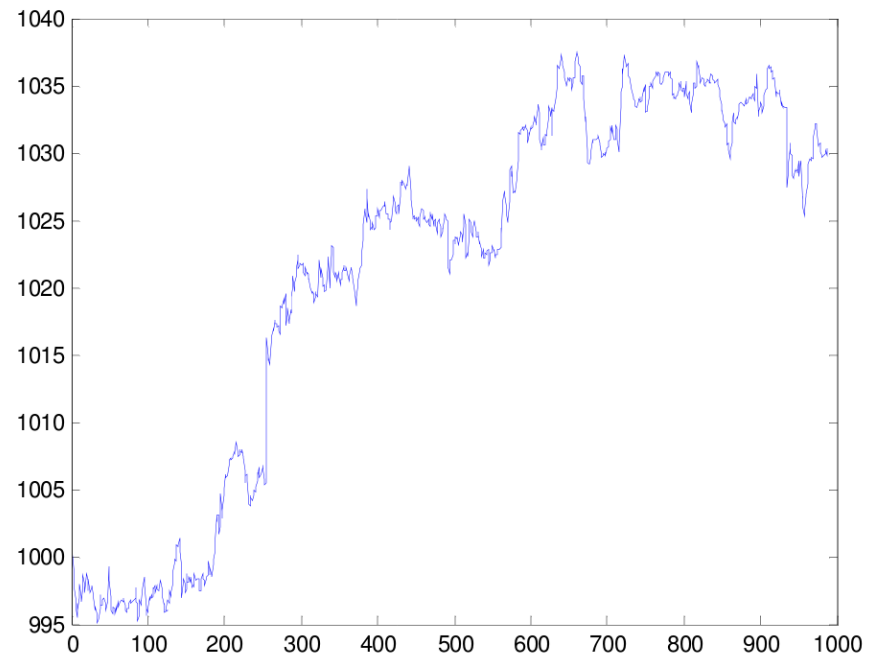

Fig. 3. Balance with respect to time

One can see that the we caan achieve a $3 \%$ or in quantity profit $=\$ 30.29 ;$ Profit $=3 \%$; MAX Drawdown $=0.5 \%$.

\subsection{Training on the mid Price and trading on Bid/Ask}

In this case we use mid-price for prediction but we trade on Bid/Ask. Here (Figure 4 and 5) on horizontal axis we have price the same number of trades as in the previous figures 2 and 3 .

The achieved profit is negative $-10.70 \%$ (Prof $-110.70 \$$ ) MAX Drawdown $=3.4 \%$.

The result is not so good as we have negative balance growth on vertical axis. However, as expected this balance is due to the fact we have not exploited the information given in the bid and ask series.

5.3 Training on the Bid/Ask and trading on the Bid/Ask In this case, we use following model to cover the spread. 


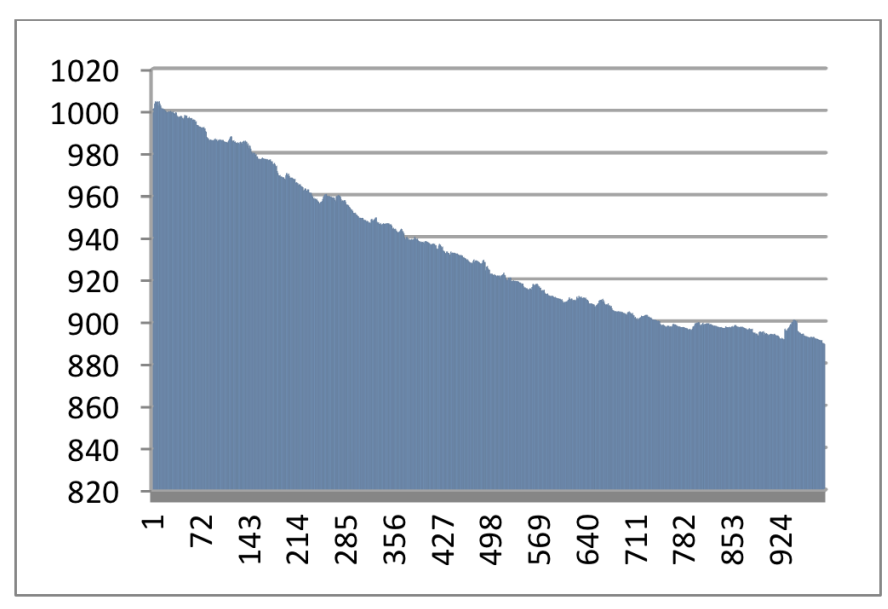

Fig. 4. Balance with respect to time

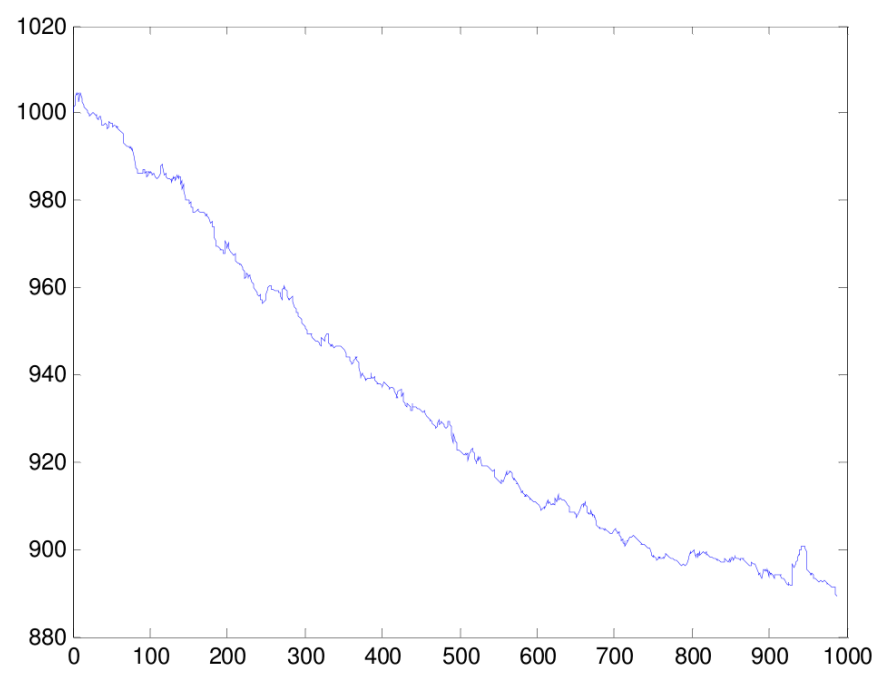

Fig. 5. Balance with respect to time

$$
\begin{aligned}
\tilde{x}_{k}=\operatorname{Net}^{(B i d)}\left(y_{k-1}, \ldots, y_{k-J}, x_{k-1}, \ldots, x_{k-J},\right. \\
\left.w_{1}, \ldots, w_{M}\right)=\operatorname{Net}^{(B i d)}(\mathbf{y}, \mathbf{x}, \mathbf{w})
\end{aligned}
$$$$
\tilde{y}_{k}=\operatorname{Net}^{(B i d)}\left(y_{k-1}, \ldots, y_{k-J}, x_{k-1}, \ldots, x_{k-J}\right.
$$$$
\left.u_{1}, \ldots, u_{M}\right)=\operatorname{Net}^{(B i d)}(\mathbf{y}, \mathbf{x}, \mathbf{u})
$$$$
\tilde{x}_{k}>y_{k-1} \Rightarrow \mathrm{BUY}
$$$$
\tilde{y}_{k}<x_{k-1} \Rightarrow \text { SELL }
$$

In the figures 6 and 7 we have smaller number of trades on horizontal axis in comparison to previous cases because it might happen that the predicted value is not greater than Ask Price or its not even less than Bid Price, therefore in some cases we do not trade. Again on the vertical axis we have account balance but this time we have some pos positive growth.

The achived profit is $1.23 \%$ (PROFIT:\$12.30)which is good in the presence of bid-ask spread. MAX Drawdown $=0.85 \%$. One can see that even in the presence of bid-ask spread the method can materialize profit.

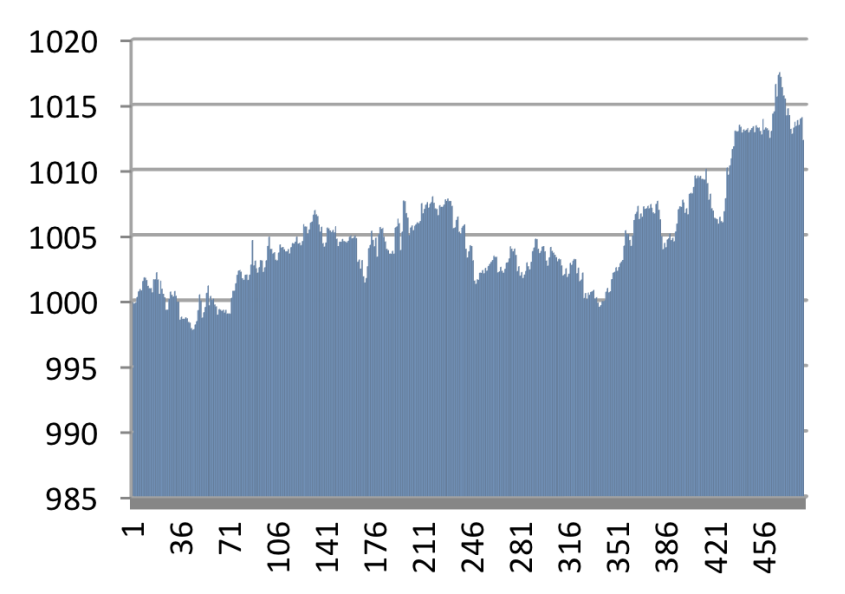

Fig. 6. Balance with respect to time

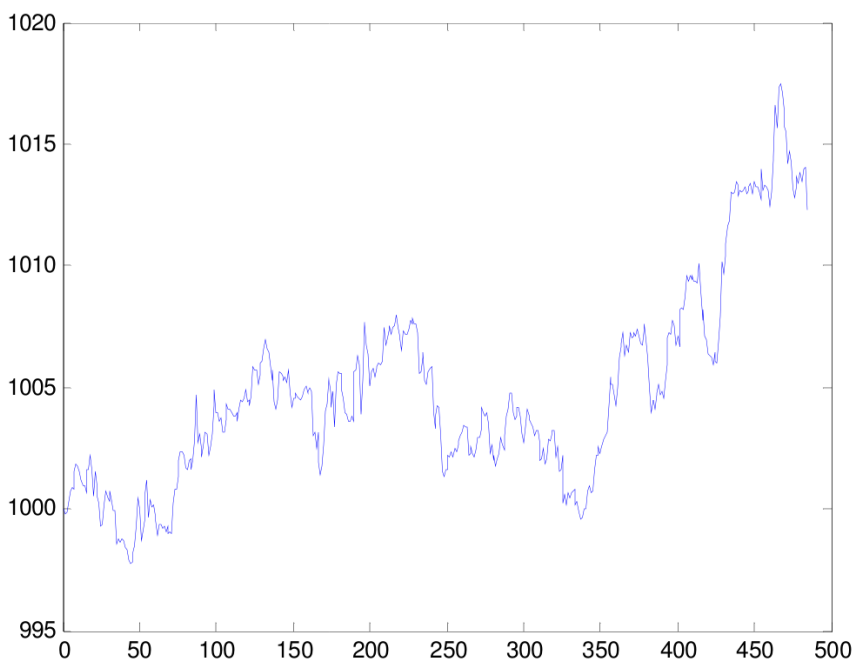

Fig. 7. Balance with respect to time

\section{Optimizing the step}

So far in our main model we only predicted the next candle, but we can also predict more than one candle. It can help us to better cover the spread and possibly extend our profit, as we let the price series change more dominantly to get out of the spread and materializing more profit. Here our goal is to find the optimal step parameter, where the step is defined as how many candles in the future we predict.

The next figure shows the result regarding the step parameter, i.e. the account profit in percentage(optimization period is 6 months) is plotted as a function of the step parameter.

From Figure 8 one can conclude that the optimal step parameter is 7 .

\section{Conclusion and further work}

In this paper we used FFNN based prediction for trading on financial time series. The ooptimal trading strategy has been derived by using the fact that FFNN can represent the conditional expected value. Furthermore, we have optimized the prediction step parameter numerically. In the case of trading on the mid- 


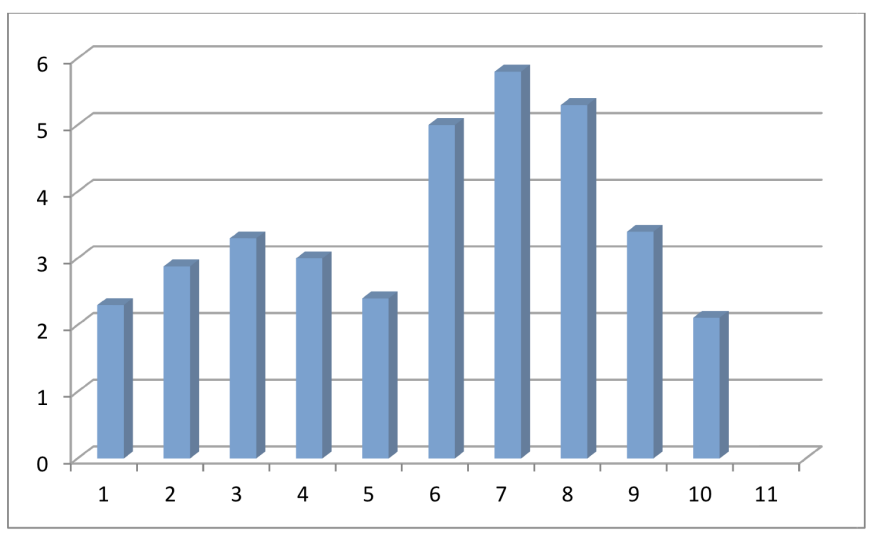

Fig. 8. Profit as a function of prediction step

price a considerable amount of profit can be accumulated. In the case of trading in the presence of bid-ask spread the method is still profitable but the achieved profit is more modest.

The methods presented here can pave the way towards high frequency, intraday trading.

As future work, one can improve the model by adding more parameters to our simple trading strategy. One parameter which we recommend is trading hours. In Forex market we have different sessions, some sessions like London and New York session are highly volatile meaning that there are bigger movements in prices. Therefore one can cover the spread more easily than in another session, so one can add this trading-hours constraint to the model to trade only in these sessions.

Furthermore, in our tests we did not use leverage, but with this low drawdowns which we had, we can easily use bigger leverages to magnify our profit. Although the ability to earn significant profits by using leverage is substantial, leverage can also work against investors. For example, if the currency underlying one of the trades moves in the opposite direction of what the investor believed would happen, leverage will greatly amplify the potential losses. To avoid such a catastrophe, forex traders usually use money management techniques.

\section{References}

1 Hanif A, Smith R E, Algorithmic, Electronic, and Automated Trading, The Journal of Trading, 7(4), (Fall 2012), 78-86, DOI 10.3905/jot.2012.7.4.078.

2 Pole A, Statistical arbitrage: algorithmic trading insights and techniques, John Wiley \& Sons Inc., Hoboken, New Jersey, 2007.

3 Kissel L R, Algorithmic Trading Strategies, Fordham University, 2006.

4 Bergan P, Devine C, Algorithmic Trading What Should You Be Doing?, Trading, 2005(1), (Spring 2005), 14-18, DOI 10.3905/tr.2005.664150.

5 Lakshman Naik R, Ramesh D, Manjula B, Govardhan A, Prediction of Stock Market Index Using Genetic Algorithm, IISTE Computer Engineering and Intelligent Systems, 3(7), (2012).

6 Zuo Y, Kita E, Up/Down Analysis of Stock Index by Using Bayesian Network, Engineering Management Research, 1(2), (2012), DOI 10.5539/emr.v1n2p46.

7 Wachter J A, Portfolio and Consumption Decisions under Mean-Reverting Returns: An Exact Solution for Complete Markets, The Journal of Financial and Quantitative Analysis, 37(1), (March 2002), 63-91, DOI $10.2307 / 3594995$.
8 D'Aspremont A, Identifying small mean-reverting portfolios, Quantitative Finance, 11(3), (2011), 351-364.

9 Balvers R, Wu Y, Gilliland E, Mean Reversion across National Stock Markets and Parametric Contrarian Investment Strategies, The Journal of Finance, 55(2), (April 2000), 745-772, DOI 10.1111/0022-1082.00225.

10 Cai L, Predictability and Hedge Fund Investing, Journal of Investment Consulting, 13(1), (2012), 30-39.

11 Kumar PN, Seshadri GR, Hariharan A, Mohandas VP, Balasubramanian P, Financial Market Prediction Using Feed Forward Neural Network, p. 77, DOI 10.1007/978-3-642-20209-4_11. 